\title{
Editorial Osteology
}

\author{
Peter Pietschmann
}

Published online: 25 August 2012

(C) Springer-Verlag Wien 2012

The field of osteology deals with the biology and pathophysiology of osseous tissue and the diagnosis and treatment of bone diseases. The spectrum of bone diseases is very broad and includes genetic, metabolic, inflammatory, malignant, and traumatic etiologies.

The first paper of this thematic issue on osteology by Reinhard Gruber very concisely summarizes the pathophysiology of bone metastases. Osteolytic or osteoblastic bone metastases are a very common complication of a variety of solid tumors such as breast or prostate cancer. Insights into the pathogenesis of bone metastases not only contribute to an understanding of the clinical manifestations but also to the mode of action of therapeutic interventions.

The next two papers deal with osteoporosis, the most frequent metabolic bone disorder. Intensive preclinical and clinical research over the past years has significantly expanded our therapeutic armentarium against this disease. Muschitz et al. review recent clinical data on the first antibody that is available for the treatment of a metabolic bone disease; denosumab, an antibody against the major osteoclast differentiation factor RANKL, has shown to be effective for the prevention of fractures in women with postmenopausal osteoporosis. Kudlacek and Puntus report on a specific form of osteoporosis: androgen deprivation therapy in men with prostate cancer is associated with a significantly increased risk of fragility fractures. The authors not only comment on the pathogenesis and epidemiology of osteoporosis in men with prostate cancer but also identify strategies for the prevention of this complication.

The final paper by Schamall et al. describes the analysis of a mediaeval skeleton with multiple injuries (probably resulting from close combat) by histology, conventional radiology and micro-computed tomography.

Assoc. Prof. P. Pietschmann, MD $(\bowtie)$

Department of Pathophysiology and Allergy Research, Center of Pathophysiology, Infectiology, and Immunology,

Medical University of Vienna,

Währinger Gürtel 18-20, 1090 Vienna, Austria

e-mail: peter.pietschmann@meduniwien.ac.at
I would like to express my sincere gratitude to all authors for contributing very interesting and timely articles. The manuscripts of this thematic issue convincingly demonstrate that the field of osteology has progressed significantly over the past years.

\section{Conflict of interest}

Dr. Peter Pietschmann has received research support and/or honoraria from: Amgen $\mathrm{GmbH}$, Eli Lilly $\mathrm{GmbH}$, Leo Pharma, Merck, Sharp \& Dohme GmbH, Nycomed Pharma, Novartis Pharma, Roche Austria, Servier Austria, Institute des Recherches Internationales Servier, Shire, Sanofi Aventis. 